\title{
Using Python to Construct a Scalable Parallel Nonlinear Wave Solver
}

\author{
Kyle T. Mandlif ${ }^{\text {đ**}}$, Amal Alghamdi ${ }^{\ddagger}$, Aron Ahmadia ${ }^{\ddagger}$, David I. Ketcheson ${ }^{\ddagger}$, William Scullin ${ }^{\S}$
}

\begin{abstract}
Computational scientists seek to provide efficient, easy-to-use tools and frameworks that enable application scientists within a specific discipline to build and/or apply numerical models with up-to-date computing technologies that can be executed on all available computing systems. Although many tools could be useful for groups beyond a specific application, it is often difficult and time consuming to combine existing software, or to adapt it for a more general purpose. Python enables a high-level approach where a general framework can be supplemented with tools written for different fields and in different languages. This is particularly important when a large number of tools are necessary, as is the case for high performance scientific codes. This motivated our development of PetClaw, a scalable distributed-memory solver for time-dependent nonlinear wave propagation, as a case-study for how Python can be used as a highlevel framework leveraging a multitude of codes, efficient both in the reuse of code and programmer productivity. We present scaling results for computations on up to four racks of Shaheen, an IBM BlueGene/P supercomputer at King Abdullah University of Science and Technology. One particularly important issue that PetClaw has faced is the overhead associated with dynamic loading leading to catastrophic scaling. We use the walla library to solve the issue which does so by supplanting high-cost filesystem calls with MPI operations at a low enough level that developers may avoid any changes to their codes.
\end{abstract}

Index Terms-parallel, scaling, finite volume, nonlinear waves, PyClaw, PetClaw, Walla

\section{Introduction}

Nowadays, highly efficient, robust, and reliable open source implementations of many numerical algorithms are available to computational scientists. However, different tools needed for a single project may not be available in a single package and may even be implemented in different programming languages. In this case, a common solution is to re-implement the various tools required in yet another software package. This approach is quite expensive in terms of effort, since a completely new code must be written, tested, and debugged. An alternative approach is to bring together the existing software tools by wrapping them in a small code based on abstractions compatible with all of them and able to interface with each programming language involved. The latter approach has the advantage that only a small amount of relatively high-level code needs to be written and debugged; the bulk of the work will still be done by the reliable, tested

\footnotetext{
* Corresponding author: mandli@amath.washington.edu II University of Washington

\$ King Abdullah University of Science and Technology

$\S$ Argonne National Labs
}

Copyright $(02011$ Kyle T. Mandli et al. This is an open-access article distributed under the terms of the Creative Commons Attribution License, which permits unrestricted use, distribution, and reproduction in any medium, provided the original author and source are credited. packages. In this paper, PyClaw and PetClaw are presented as examples of the philosophy that bridging well-established codes with high-level maintainable code is an alternative approach that leads to advantages in usability, extensibility, and maintainability when compared to completely custom built scientific software.

PyClaw and PetClaw are implemented in the Python programming language, and this choice of language has been essential to their success, for multiple reasons. Python is an interpreted scripting language that has become recognized in the scientific computing community as a viable alternative to Matlab, Octave, and other languages that are specialized for scientific work [cai2005]. For instance, Python (with the numpy package) possesses a natural and inuitive syntax for mathematical operations, has a builtin user-friendly interactive debugger, and allows simulation and visualization to be integrated into a single environment. At the same time, Python is a powerful, elegant, and flexible language. Furthermore, there exist many Python packages that make it simple to incorporate code written in $\mathrm{C}, \mathrm{C}++$, and Fortran into Python programs. Python has been suggested as particularly useful in enabling reproducible computational research [leveque2009].

\section{PyClaw and PetClaw Design and Implementation}

PyClaw and PetClaw are designed to facilitate the implementation of new algorithms and methods in the existing framework established in the well-known software package Clawpack [clawpack]. Clawpack is used to solve linear and nonlinear hyperbolic systems of partial differential equations using a Godunov type method with limiters and is written primarily in Fortran. It has been freely available since 1994 and has more than 7,000 registered users in a large variety of applications. The goal in the design of PyClaw and PetClaw is to provide interfaces to Clawpack that will facilitate the use of advanced parallel strategies, algorithm improvements, and other possible enhancements that may be field specific to the original algorithms available in Clawpack.

\section{PyClaw}

PyClaw is based on the principles of abstraction and careful definition of interfaces. These principles have allowed a broad range of extensions to be developed for PyClaw in a short period of time with wide success. The basic building blocks of PyClaw involve the separation of the data structures representing the gridded data and the domain and the solution operators that advance the gridded data to the end-time requested. Both of these abstraction layers also implement accessors that can be overridden to provide advance functionality, a feature used in PetClaw. 


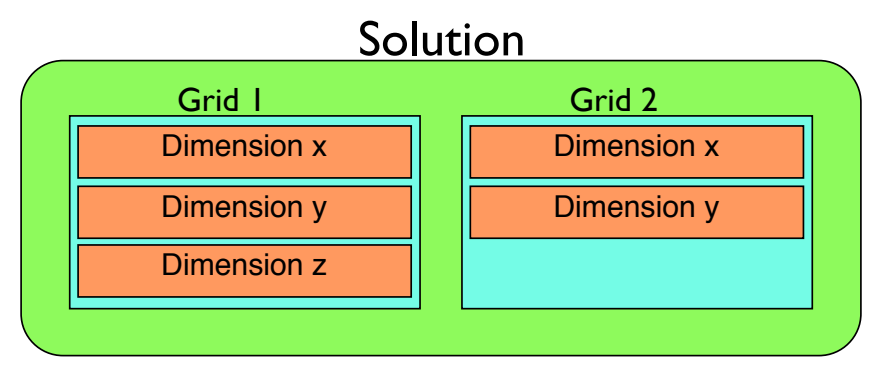

Fig. 1: PyClaw Solution structure.

The Solution class represents a snap-shot of the gridded data at a single instance in time. The class acts as a container object with possibly multiple Grid objects in the case of adaptive mesh refinement or nested grids, both of which the Clawpack algorithms are capable of. Furthermore, the Grid objects also contain a set of Dimension objects that define the domain that each Grid is defined on. Using this hierarchical class structure allows the gridded data in PyClaw to not only represent relatively arbitrarily complex gridded data but also allows individual components of the data structures to be sub-classed without the knowledge of the rest of the data structure. This is why the implementation of a package like PetClaw is as transparent as it is to the end-user. An example of a Solution object can be seen in figure 1 .

The Solver class is the numerical realization of a solution operator mapping the initial condition, represented by a Solution object, to a later time. The base Solver class defines a basic set of interfaces that must be implemented in order for the infrastructure included in PyClaw to evolve the Solution object forward in time. For instance, the routine:

evolve_to_time(solution, t)

will operate on the Solution object solution and do the necessary operations to evolve it forward in time. This is accomplished through appropriate time stepping in the base Solver object and the definition of a step() routine that the particular sub-class of Solver has implemented. This basic algorithm can be seen in figure 2 .

We expect the PyClaw code to be more easily maintainable and extensible than Clawpack, for reasons based on the difference between the Fortran 77 and Python languages [logg2010]. Fortran 77 codes generally require very long lists of arguments to subroutines, because of the lack of dynamic memory allocation and structured data types. This often leads to bugs when a subroutine interface is changed, because it is challenging to ensure that the function call is modified correctly throughout the program. In contrast, Python allows the use of extremely simple argument lists, since it has dynamic memory allocation, is object-oriented, and allows for default argument values to be pre-specified. This difference has already allowed the simple integration of different algorithms into a single framework (PyClaw). The Fortran versions of these programs share only a small fraction of code and require significantly different setup by the user, but in PyClaw switching between them is trivial.

The solvers currently available are the 2nd-order algorithms of Clawpack and the high order algorithms found in SharpClaw [sharpclaw]. Clawpack is based on a Lax-Wendroff approach plus TVD limiters, while SharpClaw is based on a method of lines approach using weighted essentially non-oscillatory (WENO) reconstruction and high order Runge-Kutta methods. The abstract

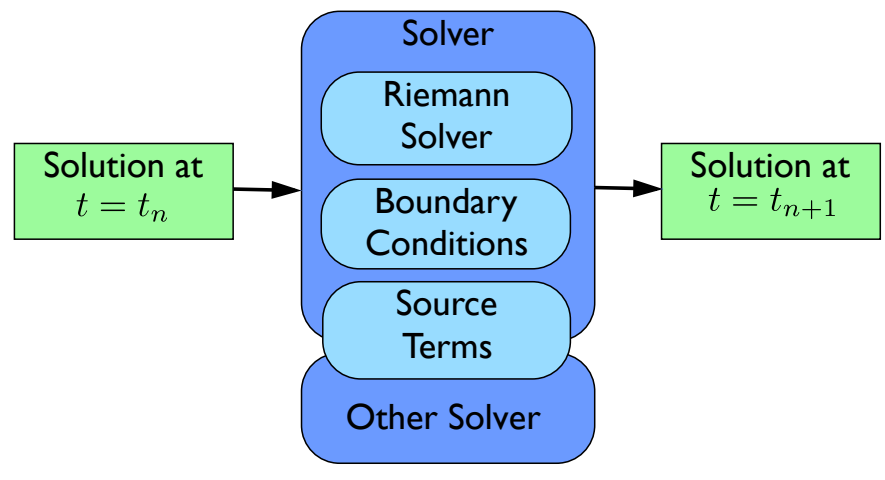

Fig. 2: PyClaw architecture flow with solver structures.

Solver class has been carefully designed to allow these solvers to be swapped trivially, i.e. by using either:

solver = pyclaw.ClawSolver2D()

for Clawpack, or:

solver $=$ pyclaw. SharpClawSolver2D()

for SharpClaw. This allows the user to easily compare the performance of different methods.

Another very useful abstraction managed by PyClaw is that of the implementation language. The 1D PyClaw solvers contain a complete implementation of both the Clawpack and SharpClaw algorithms, written entirely in Python. This is useful for rapidly prototyping, debugging, and testing modifications or new options, since new algorithms for hyperbolic PDEs are typically developed in a $1 \mathrm{D}$ setting. Since this code is written using numpy and vectorization, it is tolerably fast, but still significantly slower than compiled $\mathrm{C}$ or Fortran (vectorized numpy code is similar in speed to vectorized MATLAB code). For production runs, the user can easily switch to the more efficient wrapped Fortran codes. This is handled simply by setting the kernel_language attribute of the Solver object to "Python" or "Fortran" (the latter being the default). Even more efficient CUDA implementations of these kernels are in preparation. The benefit of this design is that the user does not need to know multiple programming languages in order to take advantage of different implementations.

\section{PetClaw}

Nilsen et. al. have suggested Python as a good high-level language for use in parallelization of scientific codes because it allows for extensive reuse of serial code and little effort (related to parallelism) from the end user [nilsen2010].

PetClaw is designed to use PETSc to add parallel functionality to PyClaw with both of these objectives in mind. This means that the (serial) PyClaw code should not need modification to accommodate PetClaw extensions and that within PetClaw all parallel operations should be handled by PETSc data structures in a way that is transparent to the user. Python makes both of these goals achievable within an elegant framework.

By implementing all necessary parallel code in Python, Nilsen demonstrated approximately $90 \%$ parallel efficiency for various applications on up to 50 processors. Because we need to go three orders of magnitude further in parallel scaling, PetClaw design goes beyond the approach suggested in [nilsen2010] and related works, by handing off all parallel operations to a widely used, robust library (PETSc) written in a compiled language. Because 


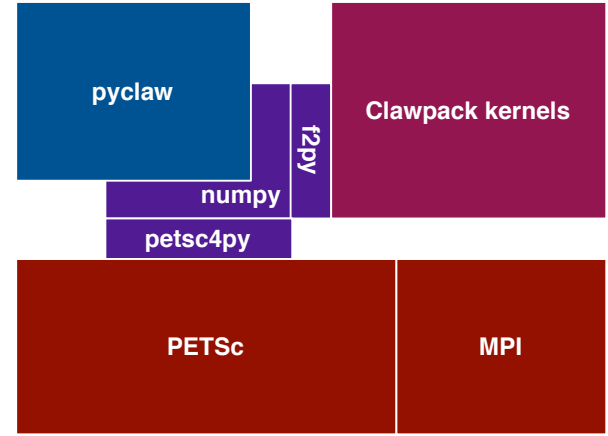

Fig. 3: Modular structure of the PetClaw code, with a focus on the orthogonality of the Fortran kernels from the parallel decomposition through PETSc.

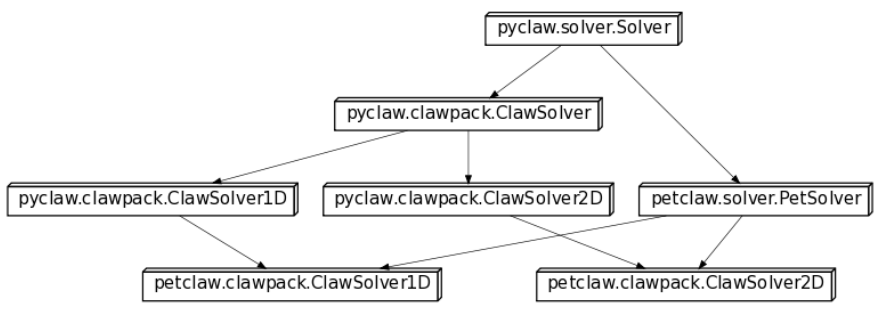

Fig. 4: Class inheritance diagram for PetClaw solvers.

PETSc is very actively developed and maintained for use in many scientific codes and on many hardware platforms, this also means that PetClaw developers don't have to worry about portability or maintenance of the parallel routines and can instead focus on the numerical algorithms that are particular to PetClaw.

An even more significant advantage gained by interfacing with PETSc that may be leveraged in the future is access to a variety of efficient parallel solvers.

Python language features and multiple inheritance have been used to make running parallel simulations with PetClaw very simple. The only difference between a serial PyClaw script and a PetClaw script that runs on tens of thousands of cores is exchanging:

import pyclaw

for:

import petclaw as pyclaw

Arrays for the solution and for coefficients that vary in space are represented by numpy arrays in PyClaw but by a custom distributed Vec class in PETSc. Using the property Python language feature, this difference is completely transparent to the user. Parallel solver classes are implemented via multiple inheritance; in most cases, a parallel solver is created merely by subclassing the corresponding serial solver as well as a base parallel solver class PetSolver; no further attributes or methods need to be implemented. As a result, the entire PetClaw extension consists of less than 300 lines of code.

Figre 4 shows how serial and parallel functionality, as well as algorithmic and dimensional differences, are implemented in an orthogonal way using class inheritance.

\section{Software Engineering}

One of the potential indirect benefits of developing a code in Python is exposure to the generally high level of software engineering practices maintained by the Python community. Primarily as a result of this exposure, PyClaw includes a suite of regression tests that currently cover $57 \%$ of the code and are being expanded. The Python package nose is used to easily run the tests or any desired subset of them. Code development is coordinated using the distributed version control software Git and the code hosting website Github. The project has an active issue tracker where bugs are reported and new features are suggested, as well as an online forum (petclaw-dev@googlegroups.com) where more detailed discussions take place. Finally, online documentation including both reference material and tutorials is maintained using the Python package Sphinx, which allows, among other things, for mathematical expressions to be included in inline code documentation and automatically rendered using LaTeX when viewed online. While many of these practices and features would be taken for granted in industrial codes, they are not standard in academic scientific codes [wilson2006].

\section{D Performance Results}

For PetClaw performance assessment with 2D problems, we have conducted on-core serial experiments to compare the performance of PetClaw code with the corresponding pure Fortran code, Clawpack. We have also performed weak scaling experiments to study the scalability of PetClaw on up to four racks of the Shaheen system. Corresponding results for PetClaw simulations in 1D may be found in [petclaw11].

\section{On-Core Performance}

We consider two systems of equations in our serial performance tests. The first is the system of $2 \mathrm{D}$ linear acoustics and the second is the $2 \mathrm{D}$ shallow water ( $\mathrm{SW}$ ) equations. The acoustics test involves a very simple Riemann solver and is intended to highlight any performance difficulties arising from the Python code overhead. The shallow water test involves a more typical, costly Riemann solver (specifically, a Roe solver with entropy fix) and should be considered as more representative of realistic nonlinear application problems.

Table 1 shows an on-core serial comparison between the Fortran-only Clawpack code and the corresponding hybrid PetClaw implementation for two systems of equations in two different platforms. Both codes rely on similar Fortran kernels that differ only in the array layout. The tests on the first platform were compiled for the x86_64 instruction set using gfortran 4.5.1 (4.5.1 20100506 (prerelease)). Each result was timed on a single core of a Quad-Core Intel Xeon 2.66GhZ Mac Pro workstation equipped with $8 \times 2$ GB $1066 \mathrm{MHz}$ DDR3 RAM. The same tests were conducted on Shaheen, on a single core of a Quad-Core PowerPC 450 processor with 4GB of available RAM. IBM XLF 11.1 Fortran compiler was used to produce a PowerPC 450d binray code in the latter platform. On both platforms, the compiler optimization flag -O3 was set. Because most of the computational cost is in executing the low-level Fortran kernels, the difference in performance is relatively minor with the difference owing primarily to the Python overhead in PetClaw. Interestingly, while the relative acoustics performance between the two codes was similar for both versions of gfortran, a significant difference was observed in the relative performance of the codes on the shallow water example, depending on the compiler version. 


\begin{tabular}{|c|c|c|c|c|}
\hline & Processor & Clawpack & PetClaw & Ratio \\
\hline Acoustics & Intel Xeon & $28 \mathrm{~s}$ & $41 \mathrm{~s}$ & 1.5 \\
\hline Shallow Water & Intel Xeon & $79 \mathrm{~s}$ & $99 \mathrm{~s}$ & 1.3 \\
\hline Acoustics & $\begin{array}{l}\text { PowerPC } \\
450\end{array}$ & $192 \mathrm{~s}$ & $316 \mathrm{~s}$ & 1.6 \\
\hline Shallow Water & $\begin{array}{l}\text { PowerPC } \\
450\end{array}$ & $714 \mathrm{~s}$ & $800 \mathrm{~s}$ & 1.1 \\
\hline
\end{tabular}

TABLE 1: Timing results in seconds for on-core serial experiment of an acoustics and shallow water problems implemented in both Clawpack and PetClaw for Intel Xeon and PowerPC 450 machines.

\section{Parallel Performance}

In our parallel performance tests, we consider the same acoustics 2D linear system used in the serial runs to represent an application where the communication over computation ratio can be relatively high due to the simplicity of its Riemann solver. We also tested 2D Euler equations of compressible fluid dynamics as a more realistic nonlinear application problem that has a relatively expensive Riemann solver.

Table 2 shows the execution time for both experiments as the number of cores increases from one core up to 16 thousand cores (four racks of BlueGene/P), with the ratio of work per core fixed. The acoustics problem used involves 178 time steps on a square grid with $160,000(400 x 400)$ grid cells per core. The Euler problem used involves 67 time steps on a grid also with 160,000 grid cells per core. The first column for each test indicates the simulation time excluding the load time required to import Python modules. The second column indicates the total simulation time, including Python module imports.

Excellent scaling is observed for both tests, apart from the dynamic loading. Profiling of the acoustics example shows that the small loss of efficiency is primarily due to the communication of the CFL number, which requires a max global reduce operation that is done each time step, and also partly due to the communication of ghost cell values between adjacent domains at each time step.

In contrast, the total job time reveals the very poor scaling of the dynamic loading time. For the largest jobs considered, this load time is roughly one hour, which is significant though generally not excessive relative to typical simulation times, since the CFL condition means that large simulations of hyperbolic problems necessarily require long run times in order for waves to propagate across the full domain. Nevertheless, this inefficiency remains as a disadvantage for high performance Python codes. Although much longer simulations can to some extent justify the start up time required for dynamic loading of Python, this loading time severely impacts parallel scaling, motivating the development of Walla to address this challenge.

\section{Addressing the Catastrophic Loading Problem with Walla}

Catastrophic scaling has been observed in applications written in all languages when they perform dynamic linking and loading on large distributed systems. Python applications are particularly prone to poor scaling due to systems issues as they tend to strongly exercise dynamic linking and loading. At the same time, Python applications provide excellent models for examining possible solutions to catastrophic dynamic link and load times [pynamic2007].

Python applications are particularly prone to poor scaling due to system overheads. They generally exercise the sort of dynamic

\begin{tabular}{lllll}
\hline & Acoustics & & Euler & \\
Cores No. & Evolve Solution & Total & Evolve Solution & Total \\
1 & 76.7 & 154 & 98.9 & 124 \\
4 & 69 & 152 & 101.1 & 123 \\
16 & 71.7 & 164 & 103.2 & 142 \\
64 & 73.7 & 217 & 103.0 & 184 \\
256 & 74 & 407 & 103.4 & 465 \\
1024 & 75 & 480 & 103.9 & 473 \\
4096 & 76.6 & 898 & 104.9 & 953 \\
16384 & 79.6 & 3707 & 112.9 & 3616 \\
\hline
\end{tabular}

TABLE 2: Timing results in seconds from scaling comparisons of the acoustics and Euler test problems for the time required for evolving the solution and the communication between processes. The total time includes the overhead due to the dynamic loading in Python and reveals the catastrophic dynamic loading problem.

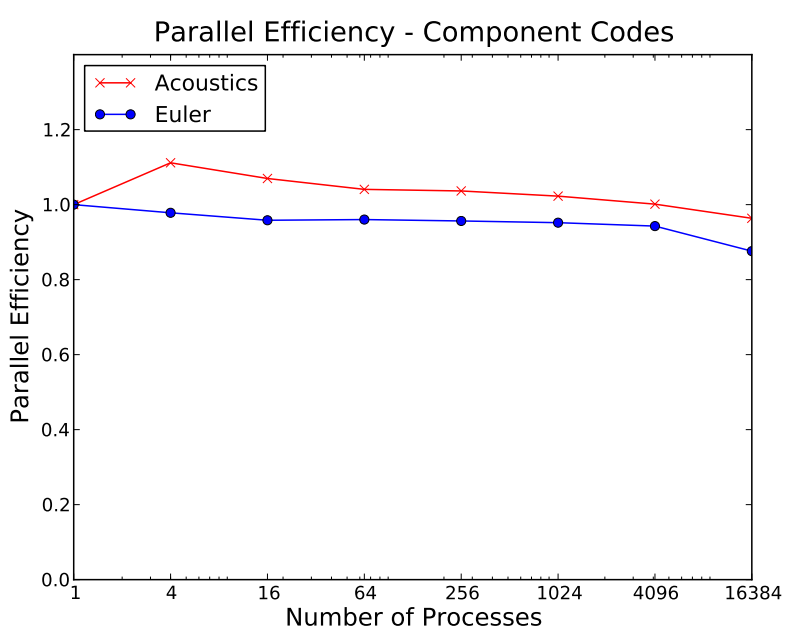

Fig. 5: Parallel efficiency results of a $2 D$ acoustics problem and a $2 D$ Euler problem for evolving the solution to the final time. These times does not include the dynamic load time of Python.

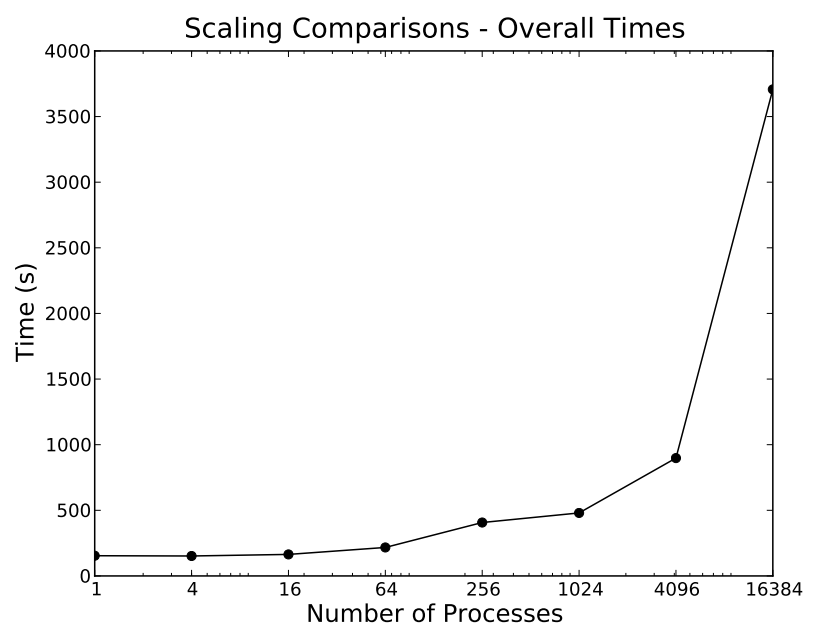

Fig. 6: Weak scaling results of the previous acoustics run from figure 5 but including the entire time to completion. 
linking and loading that creates contention for file data and metadata. In general, the farther you scale, the worse the impact on application load times becomes. This problem is well understood and benchmarks, such as in Lawrence Livermore National Laboratory's Pynamic, which help to describe and understand the extent to which an application may be impacted on a particular system [pynamic2007]. Conversely, Python applications can highlight the deficits and make it an apt platform to explore solutions.

The CPython interpreter's process for importing modules is very I/O and metadata intensive. If dynamically linked, the overhead of loading a module is further increased as Python must work through the operating system software stack before the interpreter may continue. This process is generally ignored by Python developers as single system file I/O performance is reasonable compared to the costs of computation. In large distributed systems used for scientific computation, the problem is turned on its head with file $\mathrm{I} / \mathrm{O}$, unless parallel file $\mathrm{I} / \mathrm{O}$ is available, presenting a fairly substantial bottleneck. Even where parallel I/O is available, the emphasis has been on the reading and writing of application data in a way that optimizes for file system bandwidth, generally favoring large reads and writes.

\section{Walla's Approach}

The Walla project attempts to take advantage of the high speed interconnects normally used for interprocess communication to speed dynamic loading without alteration of user codes. The project originated on IBM's Blue Gene/P platform where load times at 8,192 nodes exceeded 45 minutes for a large Python code called GPAW. Initial efforts were focused on using the low-level interface to the Blue Gene/P's high performance networks with the goal of being able to use Walla to speed all aspects of loading by coming in before the loading of MPI libraries. Due to community interest and feedback, the original codebase was abandoned in favor of using MPI for all communications ensuring portability between systems and eliminating any licensing restrictions created by use of vendor code.

In the Walla design, the CPython importer and the glibc libdl are replaced with versions that have been modified such that only a single rank performs metadata intensive tasks and file system I/O. Modifications are generally kept to a minimum with fopen and stat being replaced with versions that rely on MPI rank 0 to perform the actual fopen and stat calls, then broadcast the result to all other nodes. While wasteful of memory, the glibc fmemopen function is used to produce a file handle returned by the fopen replacement. At no time do nodes other than MPI rank 0 access Python modules or libraries via the filesystem, eliminating much of the overhead and contention that is caused by large number of ranks attempting to perform loads simultaneously.

There are a handful of caveats to using Walla. First, users must be in a situation where I/O is more expensive than broad- cast operations. While initial numbers show no significant performance hit from using Walla at small node counts, this is not guaranteed. Second, MPI_Init must already be called at the time Walla is first invoked. As Walla relies on MPI, it cannot be used to load MPI itself. The file handle generated by fmemopen does not contain and cannot be used to generate a file descriptor as the file handle is created in user space and file descriptors require the allocation of resources by the kernel. While the the handle is sufficient for use with most codes, this does create compatibility issues when an application contains calls expecting a file descriptor. Finally, some thought has to be given to the bandwidth available through
I/O networks versus the MPI broadcast otherwise it becomes easy to replace one slow loading interface with another.

Despite the need for substantial reengineering of the CPython importer internals, almost all changes should eventually be transparent to end users and require no changes to user Python codes. The runtime environment requires changes to the site.py to ensure the loading of MPI and replace the native importer with the Walla importer. For compatibility reasons, $l i b d l$ is not completely replaced; users should link libwalla before the glibc libdl to ensure that the symbols for dlopen, dlsym, and dlclose resolve back to libwalla rather than libdl.

\section{Blue Gene/P Implementation}

The Blue Gene/P platform presents additional difficulties due to I/O shipping since Blue Gene/P nodes have no local storage. At boot, operating system images get broadcasted directly into a node's memory with I/O nodes receiving a lightweight version of Linux that mounts remote file systems over a 10 Gigabit Ethernet link to a site's storage infrastructure. All Blue Gene/P nodes have three bidirectional $850 \mathrm{MBps}$ connections to a collective network designed for one-to-all high-bandwidth communications. When a compute node performs an I/O function, the operation is shipped to the I/O node via a collective network link, then processed on the I/O node, and the result returned to the compute node.

While metadata operations are easily reduced and eliminated with Walla on the Blue Gene, developers need to watch for calls that would remain local under Linux, but will be shipped on the Blue Gene such as read, seek, and close operations despite being pointed at a local memory buffer. This leads to the need to eliminate or replace read and write calls in code loading libraries and importing modules with code that directly maps or executes the contents of the broadcasted buffers. Eliminating any trace of function shipping has been a major focus of reworking the CPython importer on the Blue Gene/P platform.

\section{Conclusions and Future Directions}

We have described and demonstrated an example of using Python as the means to bind and extend the well-established hyperbolic PDE code Clawpack. The serial and parallel performance of the resulting codes are remarkable given the relatively small amount of coding (300 lines) required to turn a serial Fortran code into a scalable parallel one. This is much preferable to the alternative, more traditional approach of ex- tending legacy codes directly for high-performance computing applications using hand-coded APIs, which would be more time-consuming and more difficult to maintain.

One of the drawbacks to the approach proposed is the contention that can be caused by dynamic loading stresses on many high-performance systems. The approach introduced by Walla is a promising answer to this problem and preliminary results suggest that it may be a solution for Python codes suffering from poor scalability on distributed systems.

\section{REFERENCES}

[petclaw11] Amal Alghamdi, Aron Ahmadia, David I. Ketcheson, Matthew G. Knepley, Kyle T. Mandli, and Lisandro Dalcin. PetClaw: A scalable parallel nonlinear wave propagation solver for Python Proceedings of the High Performance Computing Symposium 2011 (2011)

[clawpack] Randall J. LeVeque, Marsha J. Berger, et. al., Clawpack Software 4.6.1, www.clawpack.org, 15 June, 2011. 
[sharpclaw] David I. Ketcheson, Matteo Parsani, and Randall J. LeVeque. High-order wave propagation algorithms for general hyperbolic systems (submitted).

[nilsen2010] J. K. Nilsen, X. Cai, B. Hoyland, and H. P. Langtangen (2010). Simplifying the parallelization of scientific codes by a function-centric approach in Python. Computational Science \& Discovery, 3, 015003.

[cai2005] Xing Cai, H. P. Langtangen, and H. Moe. On the performance of the Python programming language for serial and parallel scientific computations Scientific Programming, 13(1):31-56 (2005).

[leveque2009] R. J. LeVeque, Python Tools for Reproducible Research on Hyperbolic Problems Computing in Science \& Engineering, 11(1): 19-27 (2009).

[wilson2006] G. Wilson, Software Carpentry: Getting Scientists to Write Better Code by Making Them More Productive Computing in Science \& Engineering, 2006;8(6):66-69.

[logg2010] A. Logg, H. P. Langtangen, and X. Cai, Past and Future Perspectives on Scientific Software. In: Tveito A, Bruaset AM, Lysne O, eds. Simula Research Laboratory. Springer Berlin Heidelberg; 2010:321-362.

[pynamic2007] Gregory L. Lee, Dong H. Ahn, Bronis R. de Supinski, John Gyllenhaal, Patrick Miller, Pynamic: the Python Dynamic Benchmark IEEE Workload Characterization Symposium, pp. 101-106, 2007 IEEE 10th International Symposium on Workload Characterization, 2007. 